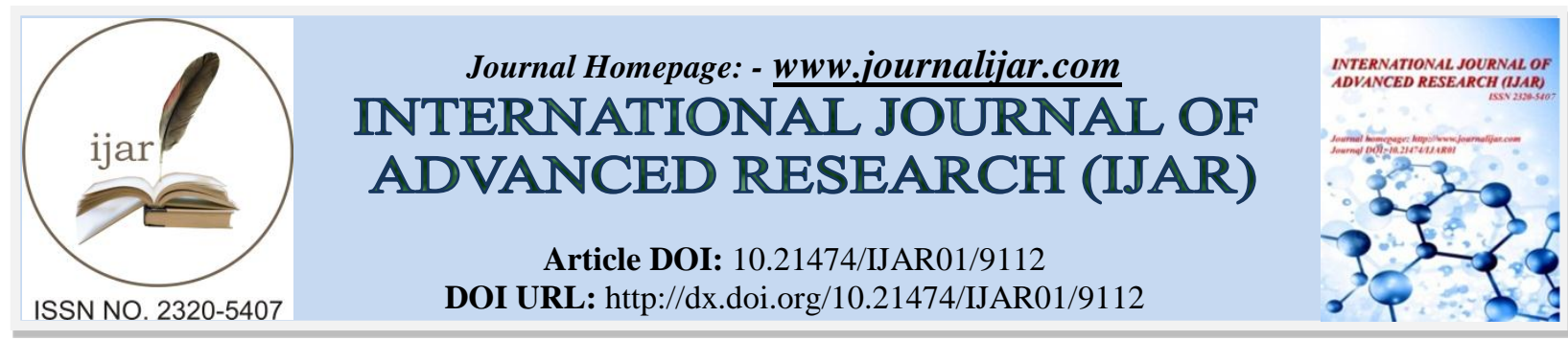

RESEARCH ARTICLE

\title{
RARE CASE OF EXTRACRANIAL SPREAD OF A RECURRENT HEMANGIOPERICYTOMA (GLOMANGIOPERICYTOMA SINONASAL TYPE) TO THE ZYGOMATICO-MAXILLARY COMPLEX: A CASE REPORT.
}

\section{Dr. Syed Sirajul Hassan, Dr. Abdulsalam Aljabab, Dr. Mahmoud AlYamany, Dr. Davide Rochetta, Dr. Hanadi.}

\section{Manuscript Info}

Manuscript History

Received: 22 March 2019

Final Accepted: 24 April 2019

Published: May 2019

\section{Abstract}

Hemangiopericytoma (HPC) is an exceedingly rare tumor of uncertain malignant potential. WHO reclassified $\mathrm{HPC}$ as a fibroblastic/myofibroblastic tumor, later discontinued the term HPC and renamed Glomangiopericytoma (GPC) ${ }^{14}$. Long-term follow up is mandatory because the histologic criteria for prediction of biologic behavior are imprecise. Glomangiopericytoma (GPC) is a rare tumor arising from the pericytes, surrounding capillaries, and accounts for less than $0.5 \%$ of all sinonasal tumors. We report a case of recurrent HPC of left maxilla removed via Weber Fergusson approach with total maxillectomy without orbital exenteration and reconstruction with Iliac and antero-lateral thigh flap for a 36-year-old man without any comorbidities, who was referred to our department from neurosurgery with recurrent massive swelling on left face with progressive exophthalmos and nasal obstruction after 2 years of pterional resection of intracranial component. The mass was well circumscribed, but diffused without any fluctuation and firm in consistency. The skin over the tumor was intact and normal. A computer tomography scan showed a large intracranial and extracranial mixed lesion in relation to left maxilla causing displacement of left orbital content but no extension. The tumor was completely removed. During surgery we found a highly vascularised tumor. The histopathologic examination revealed a cellular, highly vascularized tumor. The diagnosis was that of vascular tumor, cellular variant, with haemangiopericytoma-like features. The patient had normal postoperative course of healing and 3 months later he remains asymptomatic, without signs of recurrence or metastases.

Copy Right, IJAR, 2019,. All rights reserved.

\section{Introduction:-}

In 1942 Stout and Murray described nine tumors which were composed of capillary blood vessels with one or more layers of rounded cells arranged about them which cannot be called glomus tumors and suggested "hemangiopericytoma" (HPC) as properly descriptive name. ${ }^{11}$

Over the years, it appeared that this growth pattern was a non-specific one, shared by numerous, unrelated benign and malignant lesions, and that HPC was better considered as a diagnosis of exclusion. Three categories of lesion 
may now be individualized within the heterogeneous group of HPC like neoplasms. The first category corresponds to those non-HPC neoplasms that occasionally display HPC-like features (e.g. synovial sarcoma). Lesions belonging to the second category show clear evidence of myoid/pericytic differentiation and correspond to true HPCs. They generally show a benign clinical course, and include glomangiopericytoma/myopericytoma, infantile myofibromatosis (previously called infantile HPC), and a subset of sinonasal HPCs. The third category is the solitary fibrous tumor (SFT) lesional group, which includes fibrous-to-cellular SFTs, and related lesions such as giant cell angiofibromas and lipomatous HPCs. In practice, any HPC-like lesion can be allocated to one of these categories, leaving the ill-defined 'haemangiopericytoma' category empty. ${ }^{12}$

Glomangiopericytoma is defined as a sinonasal tumor with perivascular myoid phenotype, which accounts for less than $0.5 \%$ of all sinonasal tumors. Glomangiopericytoma was first reported as hemangiopericytoma in 1942, but this definition has been questioned. ${ }^{1}$ The World Health Organization (WHO) classified this tumor as glomangiopericytoma in $2005 .^{14}$ This classification includes the tumor described as sinonasal type hemangiopericytoma, and hemangiopericytoma-like tumor. The etiology is not clear although past trauma, hypertension, pregnancy and use of corticosteroids may be involved. ${ }^{5,14}$

\section{Case report}

First report: In 2014, a 34-year-old man with history of surgical resection of left intracranial meningioma 7 years before presented in Neurosurgical department, (King Fahad Medical City, Riyadh, KSA) with proptosis, temporal bulge on left side and right hemiparesis. Extra-ocular eye movements were normal and no problems with vision. CT Scan with contrast for Brain revealed destructive lytic lesion involving left zygomatic and lateral wall of orbit associated with soft tissue mass with heterogeneous growth. MRI revealed the same large enhancing mass. MRA and MRV showed early appearing tumor blush with arterial bleeders arising from the external carotid artery on left side and dense venous filling. Embolization of left internal maxillary artery with 6-french neuron guiding catheter and $6 \mathrm{ml}$ of Onyx was done. Angiogram revealed 70 to $80 \%$ of devascularization of tumor bed. Surgery was planned with Pterional craniotomy and resection of tumor done with primary closure. Postoperative unenhanced CT scan was done which was satisfactory with patient referred to Postoperative radiation therapy for any residual unoperable lesion. Histopathology report confirmed it as hemangiopericytoma (Glomangiopericytoma; ${ }^{5}$ HPC type $2 ;{ }^{4}$ ) Patient after discharge lost the follow up.

Second report: After 2 year's patient again reported in 2016 to neurosurgical department with a 6-month history of right nasal obstruction, decreased vision in left eye, with progressive exophthalmos. CT scan with contrast revealed tumor measuring $88 \times 75 \times 70 \mathrm{~mm}$ in diameter extending to left orbit causing proptosis, compression of extra ocular muscles and also optic nerve medially. Maxillary sinus, greater wing of sphenoid was also involved with possible extension into left nasal cavity, infratemporal fossa and masticator space. No evidence of intracranial hemorrhage noted. Multiplanar, multisequence MRI with T2 weighed imaging of brain and orbit with and without IV contrast revealed significant enlargement of the residual tumor into the left orbit, maxillary sinus, nasal cavity, maxilla with displacement of left molar teeth, masticator space and infratemporal fossa. No evidence of invasion into adjacent brain parenchyma in the middle cranial fossa. CVA and external carotid angiogram revealed that there is significant residual/recurrent left sphenoid infratemporal extra axial tumoral vascular blush supplied by significantly enlarged anterior segment of the left maxillary artery. Embolization was done with exchange of 6-French neuron guiding catheter, which was selectively navigated to left internal maxillary artery, through the guiding catheter super selective catheterization of the main feeders was performed using Apollo micro catheter. Injection of $6 \mathrm{ml}$ of Onyx was performed with nearly complete devascularization of tumor. Biopsy was done which confirmed Hemangiopericytoma (Glomangiopericytoma; ${ }^{5}$ Hemangiopericytoma type $2 ;{ }^{4}$ ). The Surgery was planned with multi disciplinary involvement of Neurosurgery, maxillofacial surgery, Plastic surgery, oculoplastic surgery, ENT teams. The surgical procedure was done under general anesthesia with nasotracheal intubation, Through the WeberFergusson approach lateral orbital extension, left total maxillectomy including orbital floor which was involved with tumor was removed enbloc with preservation of left eye, nasal mucosa, soft palate. Osteotomy extended till extracted socket of left canine. Reconstruction was initiated with left zygoma by monocortical iliac bone graft harvested from left side. Nasal mucosa along with nasopharynx was closed through primary closure. The maxillary defect was reconstructed with anterolateral thigh free flap (ALT) with a skin pedicle based on descending lateral circumflex vein perforator. ALT flap included partial vastus lateralis muscle with main pedicle was raised. Donor site closure was achieved with primary closure. The flap was placed in the defect with skin paddle forming palate and muscle filling the defect of left maxillectomy. Arteriovenous coupling was achieved with coupler size-3. Facial wound closed in layers. Blood transfusion was required intraoperatively as well as post operatively. Tracheostomy 
was done post operatively for maintenance of airway and the tracheostomy was weaned off before discharging patient home. Patient was under cover of broad-spectrum antibiotics, steroids, and analgesic during course of hospital. Patient was discharged from hospital gradually. Patient is on follow up since with good recovery of function and facial esthetics. Postoperative CT scan was taken which showed no evidence of any residual tumor. Histopathology confirmed with CD4 and immunohistochemistry confirmed to be Hemangiopericytoma (Glomangiopericytoma; ${ }^{5}$ Hemangiopericytoma type $2 ;^{4}$ ).

\section{Discussion:-}

Hemangiopericytoma is a rare vascular tumor that originates from pericytes. Hemangiopericytoma-like tumors were defined by Compagno and Hyams, as a group of tumors that develop in the head and neck region. ${ }^{13}$ They have a better prognosis despite their similarity to the conventional tumor. They often have histological features that look different from hemangiopericytoma because they express a more myogenic differentiation that resembles a glomus tumor. The term Glomangiopericytoma or sinonasal-type hemangiopericytoma is preferred to describe them.

Histologically they have a prominent pericytic vascular pattern, with thin-walled, branching vessels often with a "stag horn" configuration. However, many benign and malignant soft tissue tumors share this vascular pattern (hemangiopericytoma-like). Hemangiopericytoma is a distinct entity that exists in the original description by Stout as a tumor with a pericytic vascular pattern that lacks specific differentiation and that occurs primarily in the soft tissue of the lower extremities and retroperitoneum. ${ }^{11}$

Most of those found in the head and neck region seem to be a different histological entity from the conventional soft tissue tumor, as they have a more prominent myogenic appearance. The definition of hemangiopericytoma- like tumor has now been replaced by the term Glomangiopericytoma or sinonasal-type hemangiopericytoma, which describe a tumor thought to derive from perivascular modified smooth-muscle cells. ${ }^{5}$ The glomangiopericytoma generally shows strong immunoreactivity for muscle-specific markers such as SMA and muscle specific actin, in contrast to the conventional tumor. Our case belongs to this morphological variant. To date we could not find any tumors recurring in the maxilla without nasal extension. ${ }^{3}$

Trauma, hypertension and steroid usage are thought to be potential causes of glomangiopericytoma formation. ${ }^{1,6}$ It has a marked predilection for the nasal cavity and Para nasal sinuses although it's been reported about benign atrial glomangiopericytoma. The location such as thigh, retro peritoneum, pterygomandibular space is also known. That's why it is an equivalent term for sinonasal-type hemangiopericytoma. The term hemangiopericytoma only previously used for such type of tumors has been abandoned as they have better prognosis despite their similarity to the conventional tumors. But in our case since only the maxillary sinus without involvement of nasal cavity, so it was named still hemangiopericytoma (Glomangiopericytoma type 2 sinonasal type) since histochemistry was classical with this type. The differential diagnosis of glomangiopericytoma comprises glomangioma, myopericytoma and angioleiomyoma. ${ }^{6}$

It has a low malignant potential with excellent prognosis after surgical resection although mild nuclear pleomorphism and mitotic figures are occasionally seen.4 the survival rate achieved after surgical resection exceeds 95\%. The gold standard for treatment is resection within tumor-free margins. Endoscopic excision of tumors of the sinonasal tract has been recommended. ${ }^{1,2,6,7,8}$

The majority of finding is nasal obstruction, epistaxis, or non-specific findings, such as a mass, polyp, difficulty breathing, sinusitis and headache. Hemangiopericytoma, which is the former name of glomangiopericytoma, was defined as tumor arising from pericytes, which can occur in any location with capillaries. The diagnosis of hemangiopericytoma is often based on the architectural features, especially the capillary pattern. However, this growth pattern is non-specific, so many independent entities have been identified. ${ }^{6,7}$ The WHO unified similar concepts into the category of glomangiopericytoma in 2005. Glomangiopericytoma is defined as a sinonasal tumor demonstrating perivascular myoid phenotype, but only three cases of glomangiopericytoma have been described. ${ }^{3}$ Glomangiopericytoma is categorized as a borderline low malignancy tumor by the WHO classification. ${ }^{14}$ The local recurrence rate is reported as $16.8 \%$, but recurrence might be a consequence of incomplete initial excision, and so is better considered as residual disease. ${ }^{6}$ Thompson et al. reported no patient developed metastatic disease among 104 cases of hemangiopericytoma. However, several reports have described metastasis, including a case of liver metastasis. In our case, the tumor was completely resected so the potential for recurrence is low but the patient must 
be followed up with systemic examination. Since in our case it was recurrent so we subjected patient to postoperative radiation therapy in the first instance. ${ }^{4}$

Duval et al. revealed that although sinonasal hemangiopericytoma are more common in the middle age, they could occur in any age group, with an age range of 0 to 87 years old. A high index of suspicion is required to diagnose these tumors, as shown by the multiple case reports describing patients undergoing a polypectomy for a presumed diagnosis of nasal polyposis and the large number of patients who sought medical care on multiple occasions for epistaxis prior to reaching a diagnosis. The distinction between inflammatory polyps and hemangiopericytoma of the sinonasal cavity should be made preoperatively with a biopsy because misdiagnosis may result in an incomplete excision, which is the main risk factor leading to recurrence. Misdiagnosis may also lead to significant unexpected blood loss both intra or postoperatively. ${ }^{8}$

Imaging via CT or MRI is useful to delineate the extent of the tumor, in that it may occasionally reveal an aggressive and invasive pattern of spread that may preclude surgical resection. For larger tumors, angiography may be used to determine the vascular supply of the tumor and to allow preoperative embolization, which may decrease the blood loss at the time of surgery. Embolization was done in our case prior to surgery, which was beneficial to some extent in managing hemorrhage. , $^{8,10}$

The term "hemangiopericytoma-like tumor" has been used to describe sinonasal hemangiopericytoma (HPC) due to their seemingly innocuous behavior compared with the more aggressive behavior of HPC of soft tissue or axial skeleton. Grading of HPC remains controversial to this day, with multiple different grading schemes having been proposed. . $^{3,7,8,14}$

Radiotherapy had a high rate of recurrence or tumor-related death when used as a primary treatment modality (50\%). However, patients undergoing radiotherapy treatment following an incomplete surgical resection had a nonstatistically significant longer disease-free survival $(\mathrm{p}=0.20)^{8}$ and a statistically significant lower rate of recurrence $(\mathrm{p}=.03) .{ }^{8}$ Radiotherapy could thus represent an adjunct therapy when complete surgical resection is not possible. ${ }^{8,9}$ Long-term follow-up is required for these patients because recurrence occurs on average 46 months following initial treatment and has been reported to occur up to 17 years following initial presentation.,

Fig.1: (2014) CT with contrast and MRI axial views.

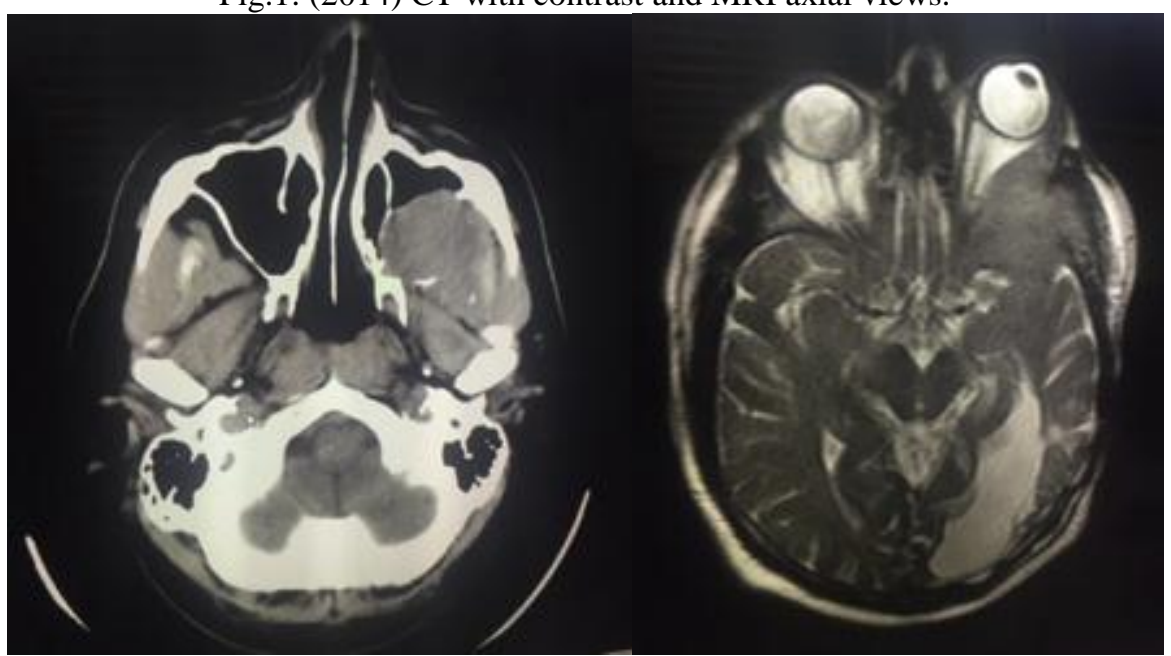


Fig.2: (2014) Embolization of Internal maxillary artery and Post resection of tumour.
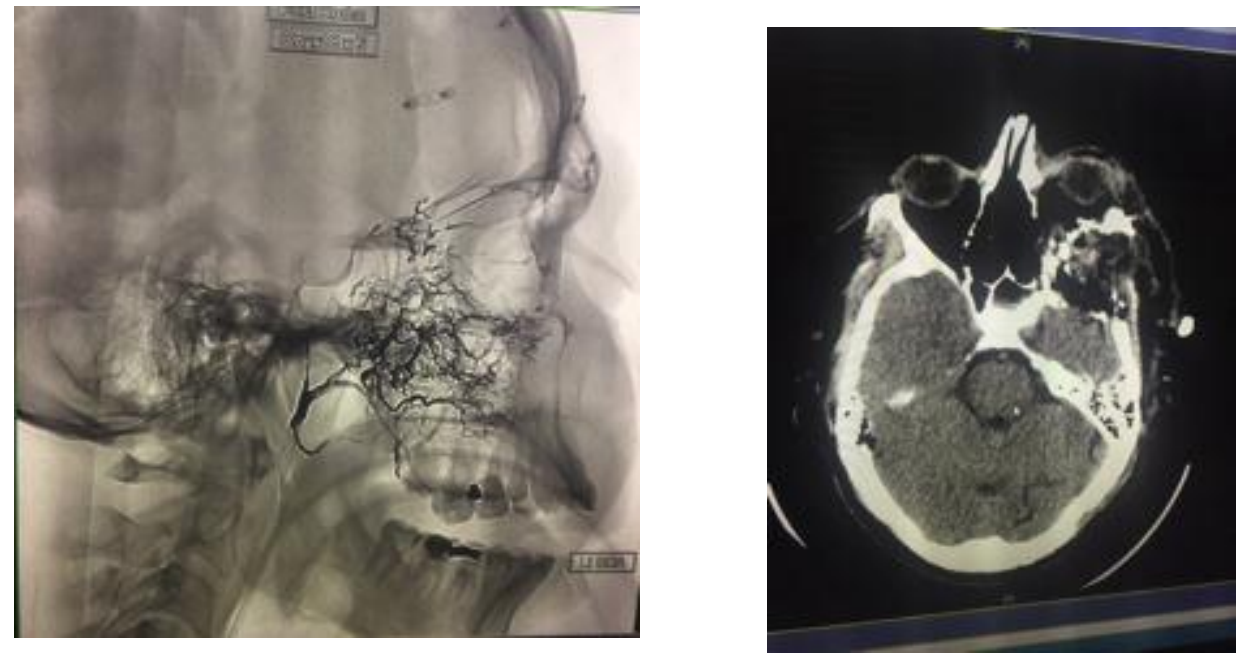

Fig.3: (2016): preoperative: Incision marking

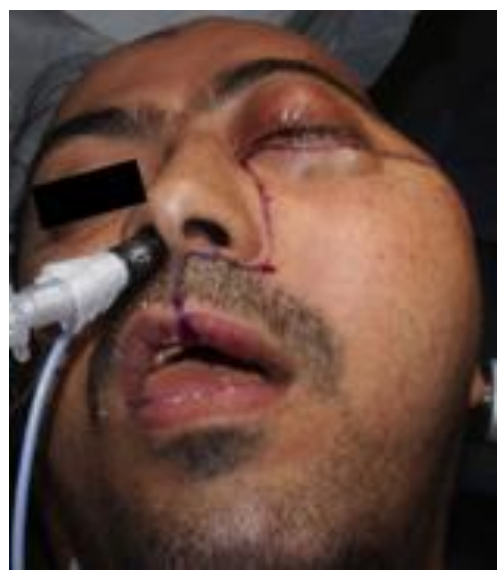

Fig. 4: (2016) MRI Images
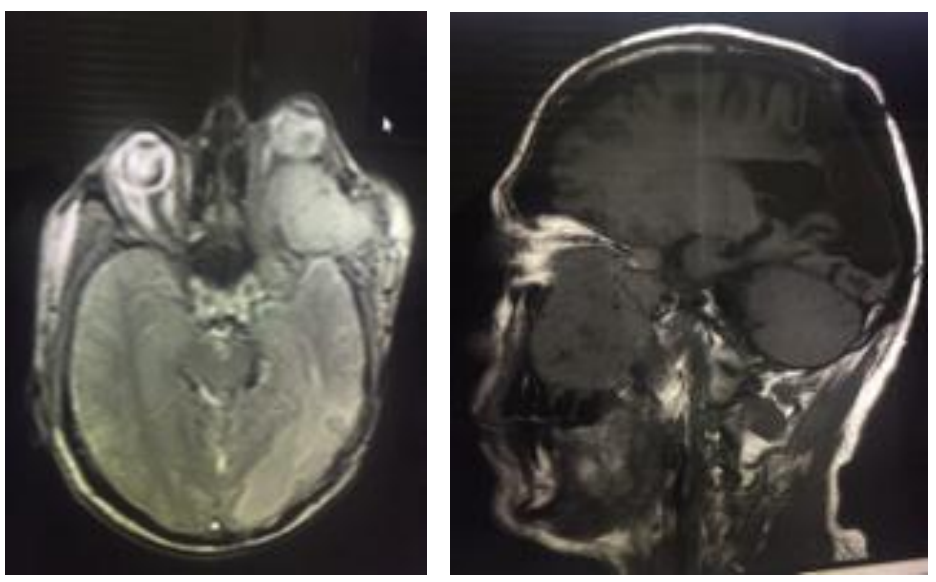

Fig.5: (2016) CT Scan and Angiogram 

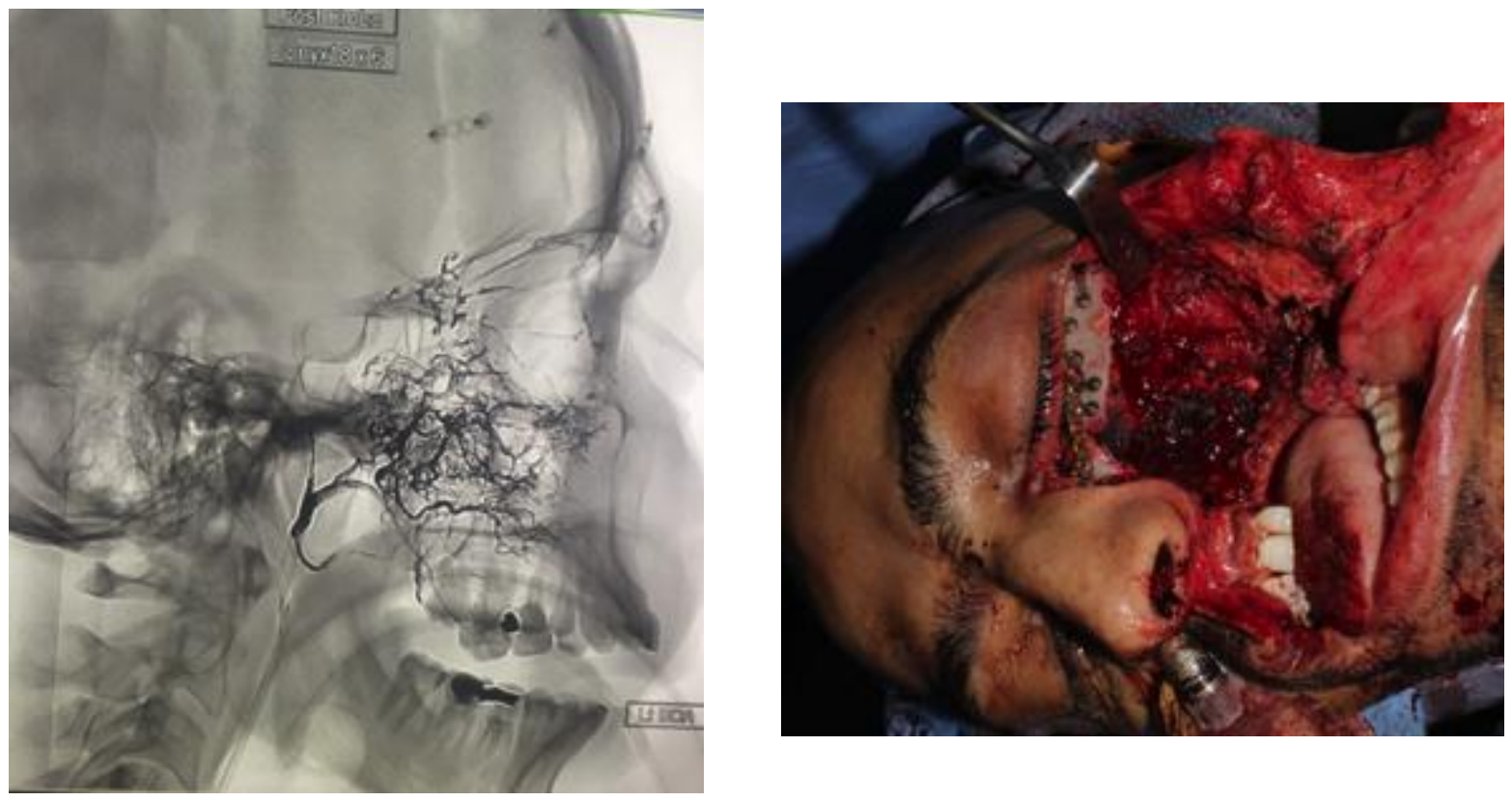

Fig.6: (2016) Intraoperative with and resected tumor.
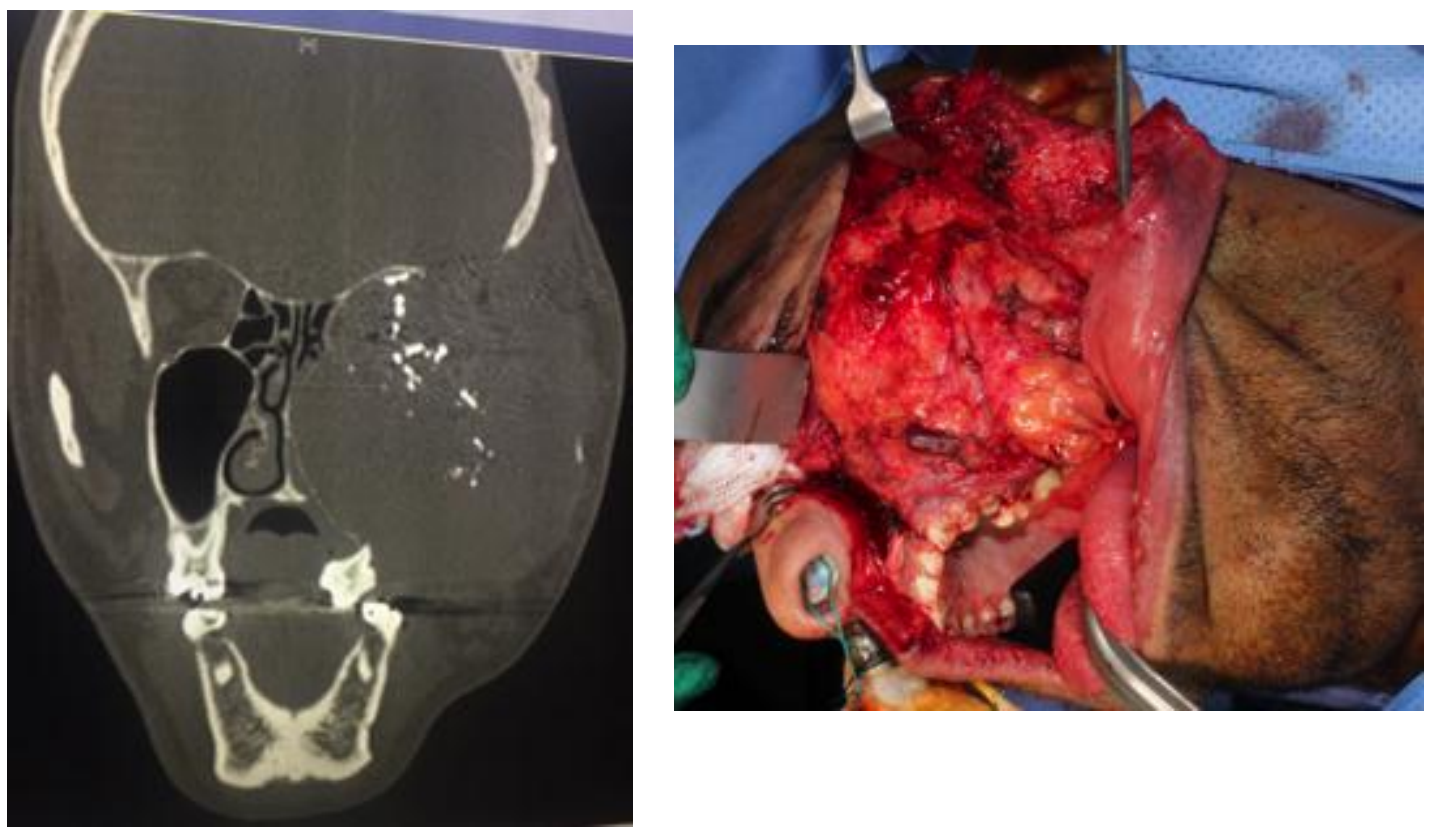
Fig. 7: (2016)
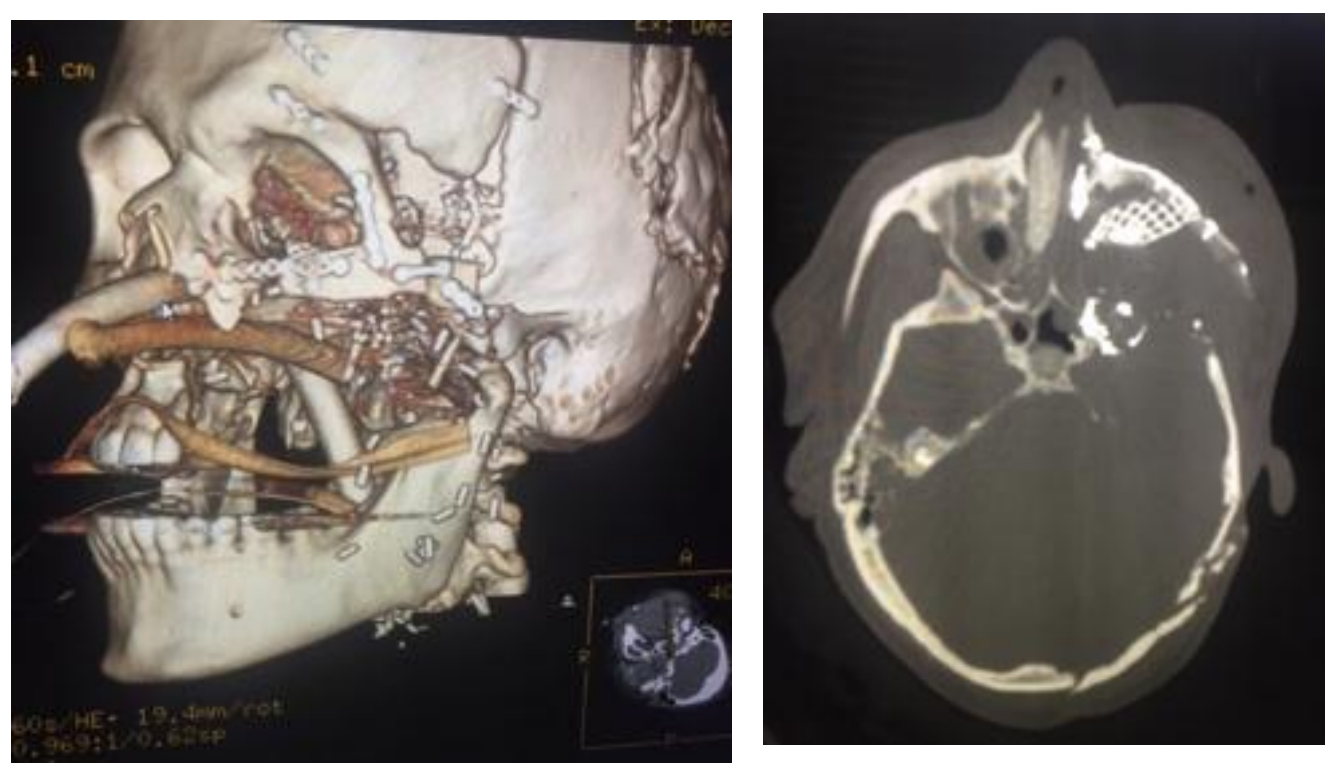

Fig. 8: (2016) Immunohistochemistry and Histology
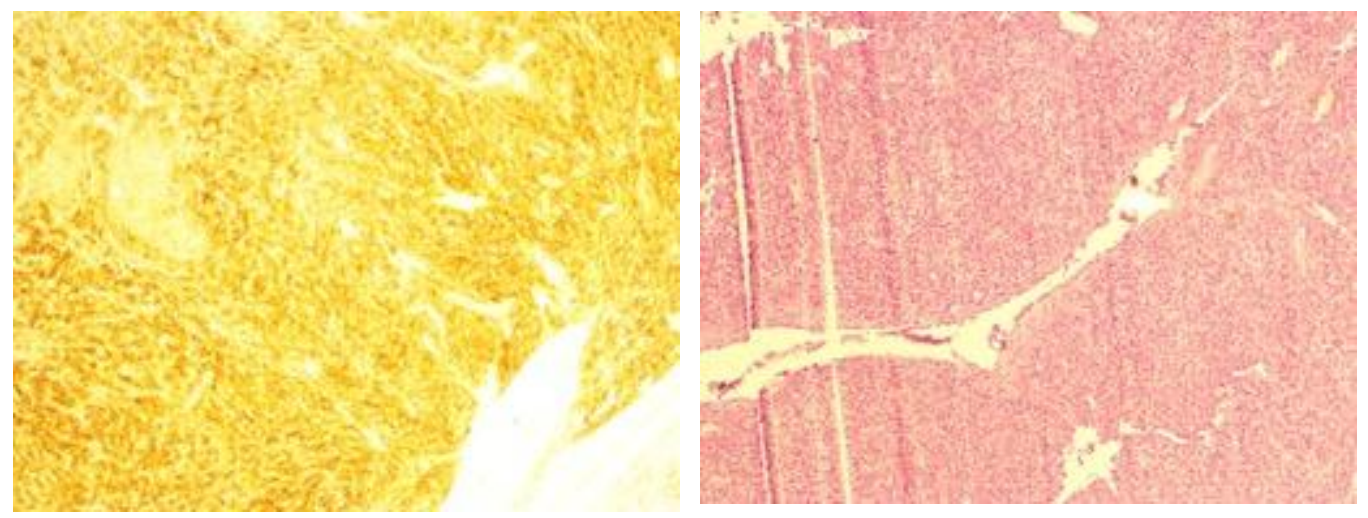

Fig.9: Postoperative 8 months
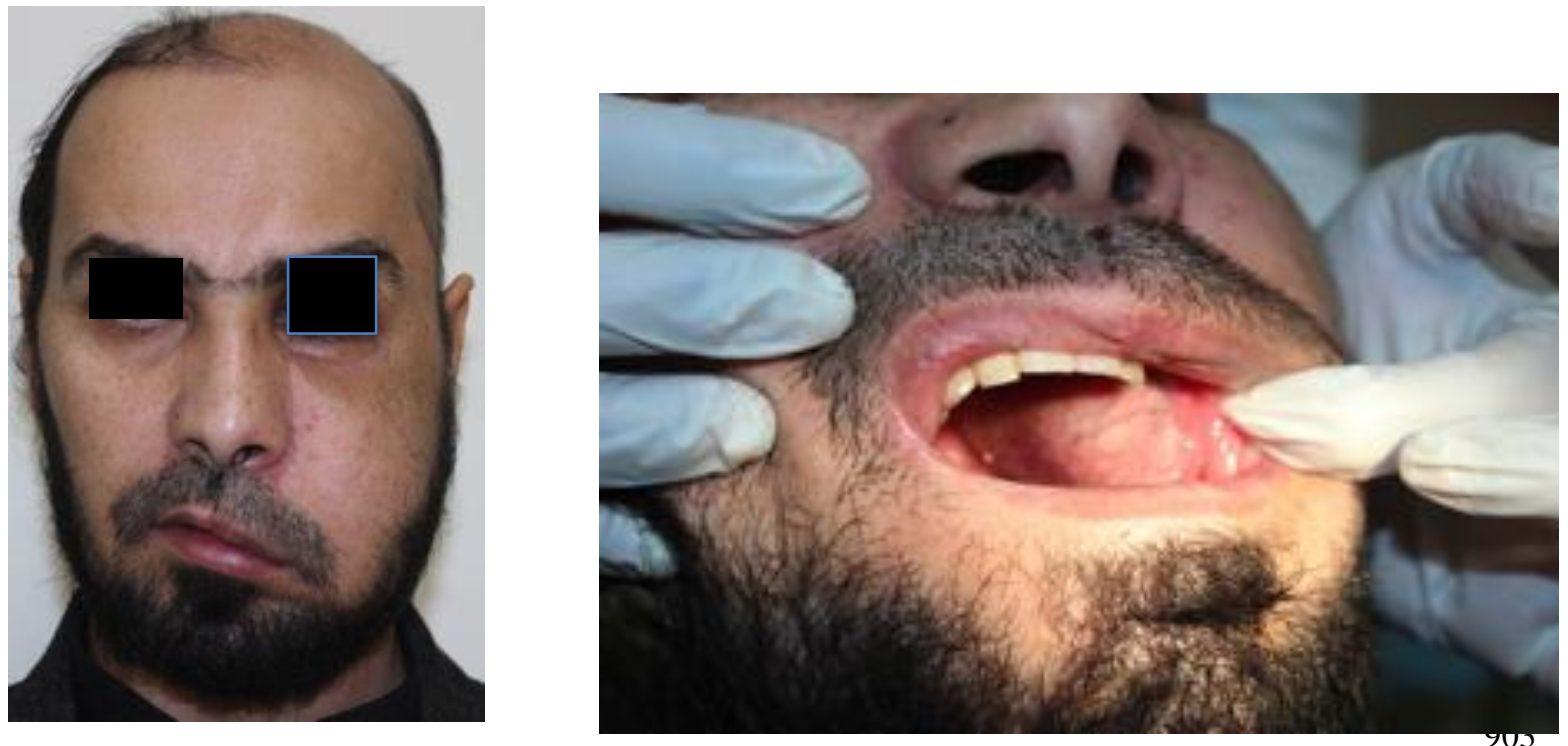


\section{References:-}

1. Chihani M, Aljalil A, Touati M, Zoubeir Y, Labraimi A, Ammar H, Bouaity B. Glomangiopericytoma: An uncommon sinonasal perivascular tumor with particular characteristics: egyptian $j$ ear nose throat and allied sci, 2011;12(3):167-70.

2. Dickson BC. An update on mesenchymal tumors of the head and neck. diagnostic histopathol, 2014;20 (8): 308-15

3. Scafati CT, D’Antonio A, Scafati ST, di Clemente SS, Parascandolo S. Glomangiopericytoma of the pterygomandibular space: An unusual case: british $j$ oral and maxillofac surg, 2007;45(12): 673-75.

4. Thompson LD, Miettinen M, Wenig BM. Sinonasal-type hemangiopericytoma: a clinicopathological and immunophenotypic analysis of 104 cases showing perivascular myoid differentiation. am $j$ surg pathol, 2003;27:737-49.

5. Higashi K, Nakaya K, Watanabe M, Ikeda R. Glomangiopericytoma of the nasal cavity. auris nasus larynx, 2011; 38: 415-17.

6. Kazmierska BZ, Grodecka J, Werfel DT, Milcarz R. The Midfacial Glomangiopericytoma A Case Report. $j$ oral biolog craniofac res, 2015; 5:124-26.

7. Thiringer JK, Costantino PD, Houston G. Sinonasal Hemangiopericytoma: Case Report and Literature Review. skull base surg, 1995; 5: 185-90.

8. Duval M, Hwang E, Kilty SJ. Systematic review of treatment and prognosis of sinonasal hemangiopericytoma. head and neck 2013; doi.10.1002:1205-10.

9. Kamath PM, Shenoy SV, Nirupama M, Raj TV. Hemangiopericytoma: A rare sinonasal tumor. egyptian j ear nose throat allied sci, 2013;14:151-54.

10. Tsirevelou P, Chlopsidis P, Zourou I, Valagiannis D, Skoulakis C. Hemangiopericytoma of the neck. head and face med, 2010;6:23.

11. Stout A, Murray MR: Hemangiopericytomas: vascular tumors arising from Zimmerman's pericytes. ann surg, 1942; 116: 26-33.

12. Gengler C, Guillou L: Solitary fibrous tumour and haemangiopericytoma: evolution of a concept. histopathol, 2006; 48: 63-74.

13. Compagno J, Hyams VJ. Hemangiopericytoma-like intranasal tumors. A clinicopathologic study of 23 cases. am j clin pathol, 1976; 66: 672-83.

14. Thompson LDR, Fanburg-Smith JC, Wenig BM. Tumours of the nasal cavity and paranasal sinuses. Borderline and low malignant potential tumours of soft tissue. In: Barnes L, et al., editors. World Health Organization (WHO) classification of tumors, pathology and genetics of head and neck tumours. Lyon: IARC Press, 2005; 43-4. 